\title{
Voltammetry of redox analytes at trace concentrations with nanoelectrode ensembles
}

\author{
Ligia Maria Moretto, Niki Pepe, Paolo Ugo* \\ Department of Physical Chemistry, Università Cà Foscari di Venezia, Calle Larga S.Marta 2137, Venezia I-30123, Italy
}

Received 24 April 2003; received in revised form 17 September 2003; accepted 22 October 2003

\begin{abstract}
Gold nanoelectrodes ensembles (NEEs) have been prepared by electroless plating of Au nanoelectrode elements within the pores of a microporous polycarbonate template membrane. Cyclic voltammograms recorded in (ferrocenylmethyl) trimethylammonium hexafluorophosphate $\left(\mathrm{FA}^{+} \mathrm{PF}_{6}{ }^{-}\right)$solutions showed that these NEEs operate in the "total-overlap" response regime, giving well resolved peak shaped voltammograms. Experimental results show that the faradaic/background currents ratios at the NEE are independent on the total geometric area of the ensemble, so that NEE can be enlarged or miniaturized at pleasure without influencing the very favorable signal/noise ratio. Differential pulse voltammetry (DPV) at the NEE is optimized for direct determinations at trace levels. DPV at NEE allowed the determination (with no preconcentration) of trace amounts of $\mathrm{FA}^{+}$, with a detection limit of $0.02 \mu \mathrm{M}$. The use of NEE and DPV in cytochrome $c$ (cyt $c$ ) solutions showed the possibility to observe the direct electrochemistry of submicromolar concentration of the protein, even without the need of adding any promoter or mediator.
\end{abstract}

(C) 2003 Elsevier B.V. All rights reserved.

Keywords: Nanoelectrodes; Cytochrome $c$; Differential pulse voltammetry; Trace electroanalysis

\section{Introduction}

Nanoelectrode ensembles (NEEs) are nanotech-based electroanalytical tools which can find application in a variety of fields [1] including electrochemical sensors [2].

Nanoelectrodes ensembles can exhibit distinct voltammetric response regimes depending on the scan rate or on the reciprocal distance between the electrode elements [1,2]. When radial diffusion boundary layers overlap totally (radius of diffusion hemisphere larger then average hemi-distance between electrodes, slow scan rates) NEEs behave as planar macroelectrodes with respect to faradaic currents [3]. The current response is dominated by radial diffusion at each single element only when diffusion hemispheres do not overlap, i.e. at high scan rates or when distances between the nanoelectrodes are large [4-6].

Martin and Menon [3] showed that NEEs behave like electrodes with partially blocked surface for which the nanodiscs area (active area) corresponds to the unblocked surface of the latter case. The theory developed by Amatore et al. [7] for

\footnotetext{
* Corresponding author. Fax: +39-041-2348594

E-mail address: ugo@unive.it (P. Ugo).
}

electrodes with partially blocked surface has been applied successfully for measuring heterogeneous electron transfer rate constants at NEEs $[3,8]$. Cyclic voltammetric responses at regular microdisc electrode arrays were also simulated recently [9], showing that there is a minimum number of elements in the array above which the normalized current response become independent on the size of the array.

As far as signal to background current ratios is concerned it was reported [2] that the cyclic voltammetric responses for a reversible redox couple at a NEE which operates in total overlap conditions, are characterized by faradaic peak currents $\left(I_{\mathrm{F}}\right)$ and capacitive background currents $\left(I_{\mathrm{C}}\right)$ given as follows:

$$
\begin{aligned}
& I_{\mathrm{F}}(\mathrm{NEE})=2.69 \times 10^{5} n^{3 / 2} A_{\text {geom }} v^{1 / 2} D^{1 / 2} c_{\mathrm{b}} \\
& I_{\mathrm{C}}(\mathrm{NEE})=A_{\mathrm{act}} v C_{\mathrm{d}}
\end{aligned}
$$

where $A_{\text {act }}$ is the active area (nanodiscs surface), $A_{\text {geom }}$ the total geometric area of the ensemble (nanodiscs plus insulator), $v$ is the scan rate, $D$ the analyte diffusion coefficient, $c_{\mathrm{b}}$ its bulk solution concentration and $C_{\mathrm{d}}$ is the double layer capacitance. 
At a conventional electrode of surface equal to $A_{\text {geom }}$, in the same experimental conditions, the same parameters obey Eqs. (3) and (4):

$$
\begin{aligned}
& I_{\mathrm{F}}(\text { conv. })=2.69 \times 10^{5} n^{3 / 2} A_{\text {geom }} v^{1 / 2} D^{1 / 2} c_{\mathrm{b}} \\
& I_{\mathrm{C}}(\text { conv. })=A_{\text {geom }} v C_{\mathrm{d}}
\end{aligned}
$$

By combining Eqs. (1)-(4), one gets:

$$
\left(\frac{I_{\mathrm{F}}}{I_{\mathrm{C}}}\right)_{\mathrm{NEE}}=\left(\frac{I_{\mathrm{F}}}{I_{\mathrm{C}}}\right)_{\text {conv }} \frac{A_{\text {geom }}}{A_{\text {act }}}
$$

Eq. (5) puts in evidence that the signal/background current ratio at the NEE is higher than the signal/background current ratio at a conventional electrode of the same geometric area for a proportionality factor that is the reciprocal of the fractional electrode area $f$, defined as

$f=\frac{A_{\text {act }}}{A_{\text {geom }}}$

In theory, this ratio should be independent on the overall geometric dimension of the ensemble.

Experiments showed that for $f$ values between $10^{-3}$ and $10^{-2}$ detection limits at NEEs were $2-3$ orders of magnitude lower than with conventional electrodes $[3,8,10]$. However, NEEs of rather large geometric area (typically $0.07 \mathrm{~cm}^{2}$ ) were used in these experiments.

For a fixed $A_{\text {geom }}$ value, the voltammetric signal at a NEE is maximum when a total overlap regime is operative, being lower in the case of a pure radial regime. In the latter case, in fact, only a certain percentage of the geometric area of the ensemble contributes to produce a faradaic current while, in the total overlap regime this percentage is $100 \%$. Note that the Faradaic currents at NEEs in total overlap regime are identical to those at conventional electrodes of the same geometric area as the ensemble and that the improvement produced by the use of the NEE is all in the dramatic lowering of capacitive current.

The ability of NEEs to furnish well resolved cyclic voltammograms for trace redox species is particularly attractive for analytical purposes since, in principle, it allows the electrochemical detection of low analyte concentrations avoiding the use of tedious and time consuming preconcentration steps (both faradic and non-faradic). This seems particularly interesting for direct "in field" analysis for in-real-time environmental monitoring and for "in situ" and "in vivo" electroanalysis in biological samples. Also the possibility to lower the overall NEE dimension while keeping unaltered the signal/background ratio is attractive for NEEs miniaturization and use in small volume electrochemical cells.

The present paper reports the results of a study aimed to examine the dependence of typical analytical parameters such as faradaic current/background current ratios, sensitivities and detection capabilities of NEEs as a function of geometric factors involved in the fabrication of these electrode systems as well as the improvements eventually achievable by combining the use of NEEs with differential pulse voltammetry (DPV). Target analytes used to show these possibilities are reversible "simple" redox probes such as the (ferrocenylmethyl)trimethylammonium cation as well as more complex redox systems like the redox protein cytochrome $c$ (cyt $c$ ).

\section{Experimental}

\subsection{Chemicals and reagents}

Polycarbonate filtration membranes (SPI-Pore, $47 \mathrm{~mm}$ filter diameter, $6 \mu \mathrm{m}$ filter thickness) having a nominal pore diameter of $30 \mathrm{~nm}$, a nominal pore density of $6 \times 10^{8}$ pores $\mathrm{cm}^{-2}$ and coated with the wetting agent polyvinylpyrrolidone were used as the templates to prepare the NEEs. Commercial gold electroless plating solution (Oromerse Part B, Technic Inc.) was diluted (40 times with water) prior to use. (Ferrocenylmethyl)dimethylamine (Aldrich) was reacted with methyl iodide to form the quaternary ammonium iodide [11]. This was then converted to (ferrocenylmethyl)trimethylammonium hexafluorophosphate $\left(\mathrm{FA}^{+} \mathrm{PF}_{6}^{-}\right.$) using $\mathrm{AgPF}_{6}$.

Horse heart cytochrome $c$ (type VI, molecular weight $12,384)$ was purchased from Sigma and used without any further purification. Solution concentrations of cytochrome $c$ were quantified by UV-Vis absorption spectroscopy $\left(\varepsilon_{(\mathrm{CytC}-\mathrm{Fe}(\mathrm{II}), 550 \mathrm{~nm}}=2.95 \times 10^{4} \mathrm{M}^{-1} \mathrm{~cm}^{-1}[12]\right) ;$ they agreed with the concentration values expected on the basis of the molecular weight given by the supplier. Note that both on the basis of our experience [13] and on previous literature report $[14,15]$ voltammetric responses obtained on this kind of commercial preparation do not differ significatively from those of purified samples.

All other reagents were of analytical grade and were used as received. Purified water was obtained using a Milli-Ro plus Milli-Q (Millipore) water purification system.

\subsection{Instruments}

All electroanalytical measurements were carried out at room temperature $\left(22 \pm 1{ }^{\circ} \mathrm{C}\right)$ using a three-electrodes single-compartment cell (volume $2 \mathrm{ml}$ ) equipped with a platinum coil counter electrode and an $\mathrm{Ag} / \mathrm{AgCl}(\mathrm{KCl}$ saturated) reference electrode. All potential values are referred to this reference electrode. A CH620A apparatus controlled via PC by their own software, was used for voltammetric measurements.

\subsection{Preparation of the electrodes}

The nanoelectrode ensembles were prepared using the electroless plating procedure described previously [3] and following modifications [8]. 
Also the final assembly of the NEE for obtaining electrodes handy for use in an electrochemical cell followed substantially the previous method [3], however slightly modifying the final assembly in that, the copper tape, which acts as electrical connection for the NEE was attached to the lower gold layer which completely covers one face of the membrane, instead of being attached to the upper gold layer as previously done [3]. This modification improved the electrical connection between copper and the NEE.

SEM and TEM analyses of these NEEs [16] allowed us to estimate a values of $f=A_{\text {act }} / A_{\text {geom }}=(1.5-2.0) \times 10^{-2}$.

The geometric area of the NEE is defined by the diameter of a hole punched in the insulating tape which covers the upper face (peeled) of the NEE. Holes diameters were: 1, 3 (typical value), and $8.6 \mathrm{~mm}$.

Conventional "macro" gold disk electrodes (area = $0.07 \mathrm{~cm}^{2}$, sealed in Teflon) were polished to a mirror finish on a polishing cloth before use; for brevity, these electrodes are named "Au-macro" hereafter.

\section{Results and discussion}

\subsection{Cyclic voltammetric characterization}

Cyclic voltammograms (CVs) recorded at the NEEs used in this work in $5 \mu \mathrm{M} \mathrm{FA}^{+}\left(10^{-3} \mathrm{M} \mathrm{NaNO}_{3}\right.$ as the supporting electrolyte) showed peak shaped diffusion controlled patterns since $I_{\mathrm{p}}$ depends linearly on $v^{1 / 2}$ [17] (where $I_{\mathrm{p}}$ is the faradaic peak current). Other characteristics of the CVs were comparable with previous reports [3,8]; in particular, the capacitive currents were significantly lower than those observed at macro-electrodes of the same geometric area.

A feature which was not yet examined is the influence of the total geometric area of the ensemble on the faradaic/background current ratios. As shown in Fig. 1, CVs recorded at different scan rates at NEEs of different geometric areas (namely, $0.008,0.071$ and $0.580 \mathrm{~cm}^{2}$ ), but with constant values for the fractional areas, showed that faradaic peak currents scale with the geometric area, as background capacitive currents do, but the ratio between these parameters, at a fixed scan rate, remains independent on $A_{\text {geom }}$. The ratios $I_{\mathrm{p}} / A_{\text {geom }}$, depend linearly on the square root of the scan rates in the range $2-100 \mathrm{mV} \mathrm{s}^{-1}$, with slopes that are practically equal to each other. The average value for the quantity $I_{\mathrm{p}} A_{\text {geom }}{ }^{-1} v^{-1 / 2}$ for these NEEs is equal to $2.8 \pm 0.2 \mu \mathrm{Acm}^{-2} \mathrm{~V}^{-1 / 2} \mathrm{~s}^{1 / 2}$. At a scan rate of $0.02 \mathrm{~V} \mathrm{~s}^{-1}$ for these NEEs, peak current density, normalized over the concentration and calculated as $I_{\mathrm{p}} A_{\text {geom }}{ }^{-1} c_{\mathrm{b}}{ }^{-1}$, is $79 \pm 4 \mathrm{nA} \mathrm{cm}^{-2} \mu \mathrm{M}^{-1}$. If the geometric area (nanodiscs + insulator) is substituted with the active area (nanodisks surface) this value increases 40 times (which correspond to the reciprocal of the fractional electrode area) and becomes $3.16 \pm 0.16 \mu \mathrm{Acm}^{-2} \mu \mathrm{M}^{-1}$.

The evidence that the resolution of signal from background currents is independent on the total area of the en-
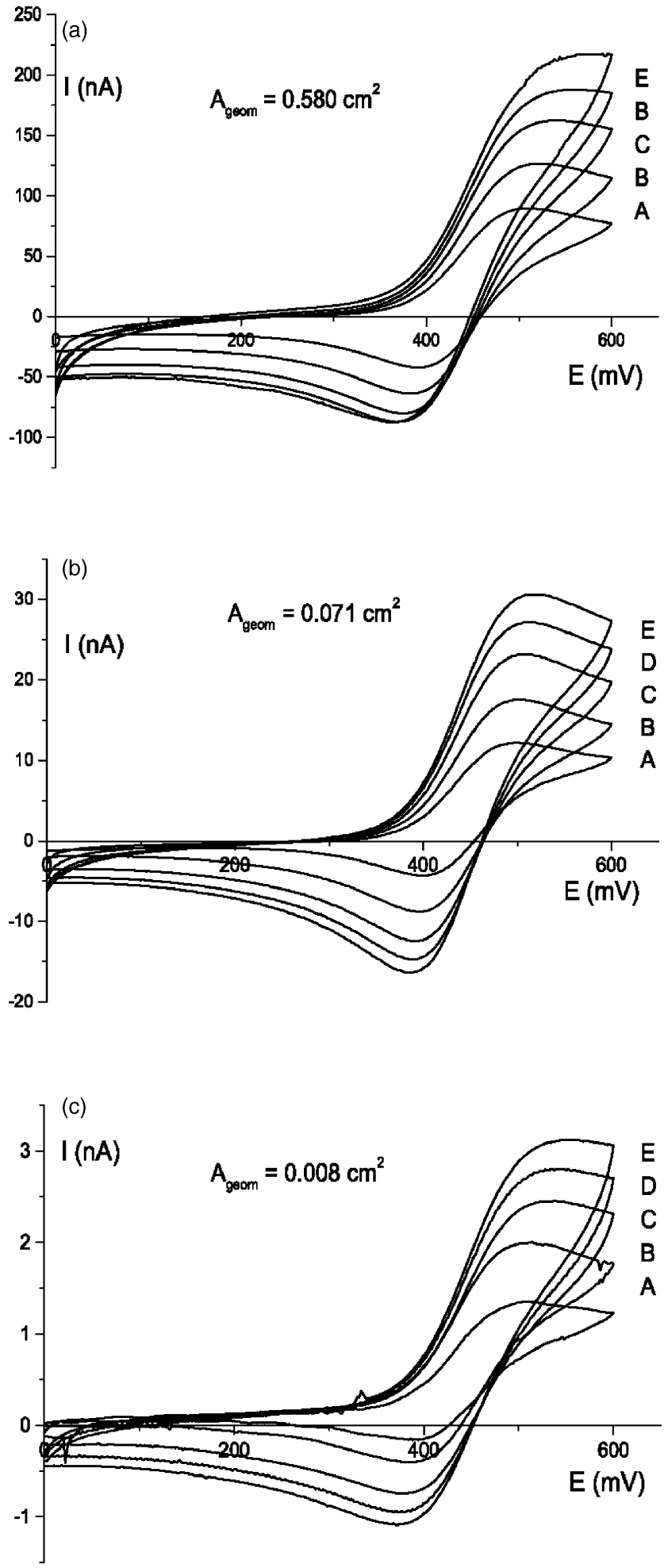

Fig. 1. Cyclic voltammograms recorded at NEEs with different geometric areas: (a) $0.580 \mathrm{~cm}^{2}$; (b) $0.071 \mathrm{~cm}^{2}$ and (c) $0.008 \mathrm{~cm}^{2}$, in $5 \mu \mathrm{M}$ $\mathrm{FA}^{+} 10^{-3} \mathrm{M} \mathrm{NaNO}_{3}$ solution at different scan rates: (A) $2 \mathrm{mV} \mathrm{s}^{-1}$; (B) $5 \mathrm{mV} \mathrm{s}^{-1}$; (C) $10 \mathrm{mV} \mathrm{s}^{-1}$; (D) $15 \mathrm{mV} \mathrm{s}^{-1}$ and (E) $20 \mathrm{mV} \mathrm{s}^{-1}$. 
semble, indicates that enlarging or reducing the $A_{\text {geom }}$ of these NEEs within the dimensional limits indicated in Fig. 1 has no practical influence on the signal characteristics. Literature results obtained by the simulation of CVs at ultramicroelectrode arrays [9] indicate that such an independence is observed when the number of active elements in an array/ensemble approaches infinite. This appears to be the case even for the ensemble with smallest geometric area used by us (namely, $0.008 \mathrm{~cm}^{2}$ with about $4.8 \times 10^{6}$ nanodiscs).

\subsection{Differential pulse voltammetry with NEEs for trace analyses}

In order to improve signal/noise ratios and, consequently, to achieve lower detection limits, pulsed electroanalytical techniques are commonly used [17]. As shown above and on the basis of previous experimental results, NEEs show properties which allow one to achieve already a significant lowering of capacitive currents thanks to their geometrical and diffusive characteristics. It appears therefore interesting to examine whether the use of a pulsed technique, such as DPV, can further improve the analytical performances of NEEs.

Fig. 2 shows DPV patterns recorded at a NEE (Fig. 2a) and at a Au-macro (Fig. 2b) in solutions containing
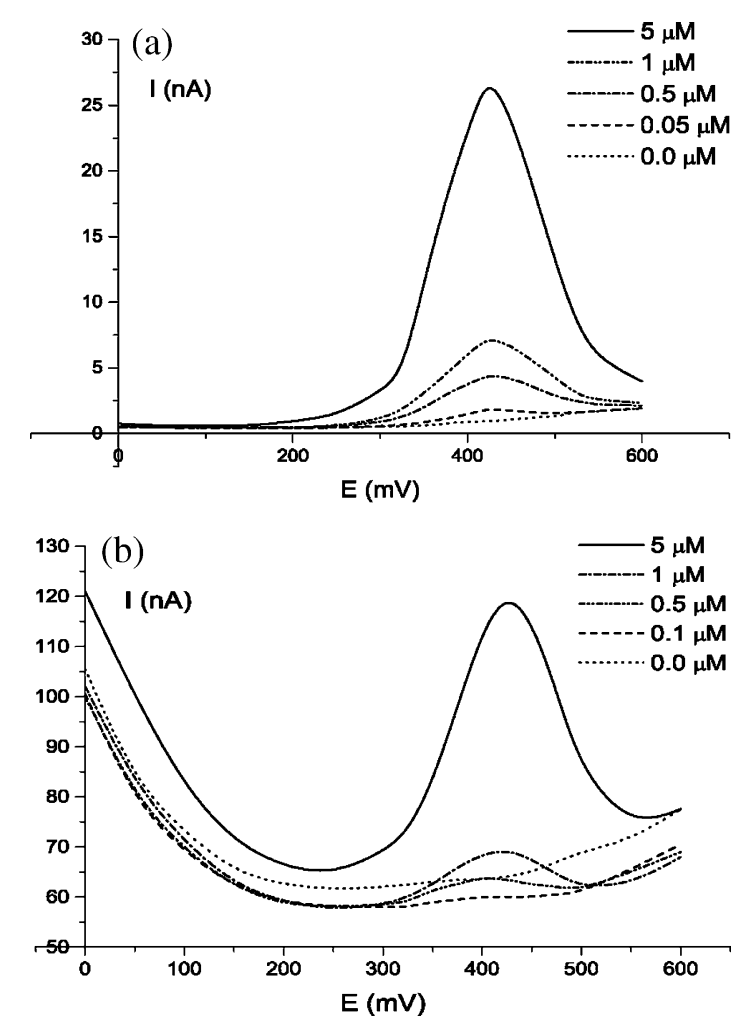

Fig. 2. Differential pulse voltammograms recorded at NEEs (a) and Au macroelectrode (b), in $10^{-3} \mathrm{M} \mathrm{NaNO}_{3}$ solutions containing increasing $\mathrm{FA}^{+}$concentration, as indicated in the figure. Experimental parameters: scan rate $5 \mathrm{mV} \mathrm{s}^{-1}$, pulse height $25 \mathrm{mV}$, pulse time $50 \mathrm{~ms}$, initial potential $0 \mathrm{mV}$ and final potential $+600 \mathrm{mV} ; A_{\text {geom }}=0.071 \mathrm{~cm}^{2}$. (sub)micromolar concentrations of $\mathrm{FA}^{+}$. At variance with the CV case [3,8], DPV peak currents (faradaic currents) at the NEE are slightly lower than those at the Au-macro. This is related to the smaller time scale of the DPV experiments, this parameter being equal to the pulse time for such a technique (namely, $50 \mathrm{~ms}$ for DPV in the experimental conditions of Fig. 2 versus $1300 \mathrm{~ms}$ for CV, e.g. at $20 \mathrm{mV} \mathrm{s}^{-1}$ ) [17]. A shorter time scale can cause, in fact, the transition from total overalp to partial overlap conditions in the ensemble [4-6]; in the latter case less than $100 \%$ of $A_{\text {geom }}$ contributes to the faradaic current. This notwithstanding, the sensitivity $(m)$ of DPV peak currents at NEEs (given as the slope of the linear calibration plot, not shown), is still high being equal to $7 \mathrm{nA} / \mu \mathrm{M}$. Another analytically important characteristic which distinguishes NEEs from macroelectrodes is that the background noise is significantly lower for the nanoensemble even using DPV. The voltammograms in Fig. 2a put in evidence that at the NEE, the background current is almost a flat line which favors the easy resolution of the peak current signal from the background currents, even at the lowest concentrations examined here (namely $5 \times 10^{-8} \mathrm{M}$ ). On the contrary, at the macroelectrode, at high amplification levels, background currents show a typical parabolic shape which hinders the detection of small peak currents. This can be the basis for further advances in the analytical uses of NEEs, in particular for the determination of analytes which are electroactive at very positive or very negative potential values (i.e. in the proximity of the cathodic or anodic limits of the accessible potential window).

The improvement of background signals related to the use of NEEs is quantified by the measurement of the background noise $\left(s_{\mathrm{b}}\right)$, which is calculated as the standard deviation of five independent blank measurements and results 0.05 and $1.0 \mathrm{nA}$ for NEE and Au-macro, respectively. Detection limits (DLs) for $\mathrm{FA}^{+}$, obtained as $3 s_{\mathrm{b}} m^{-1}$, are $0.02 \mu \mathrm{M}$ for NEE and $0.42 \mu \mathrm{M}$ for the Au-macro. Note that DL with NEEs is about 20 times lower than with the Au-macro and not 40 times lower as expected simply on the basis of Eqs. (5) and (6). This agrees with the above reported considerations on the influence of the shorter time scale of DPV on the diffusive regime at NEEs. In any case DPV at NEEs is still able to lower DL of more than one order of magnitude with respect to DPV on Au-macro.

In order to check the possibility to use DPV at NEEs for trace determinations even of those analytes which are "difficult" to determine, a series of tests were performed in solutions containing the redox protein cytochrome $c$. Really, the electrochemistry of cyt $c$ has been studied widely; however the usual procedure to obtain the protein electrochemistry signal is to add to the cyt $c$ solution a suitable promoter (typically $4,4^{\prime}$-bipy $[18,19]$ or $4,4^{\prime}$-dipirydil sulfide $[20,21]$ or others $[22,23]$ ) or to use electrodes modified with polyanionic coatings or membranes [13,24]; in both cases the modifier interacts with the protein and orient it in a way which favors the electron transfer. A couple of examples showing the possibility to avoid the use of the promoter has been 


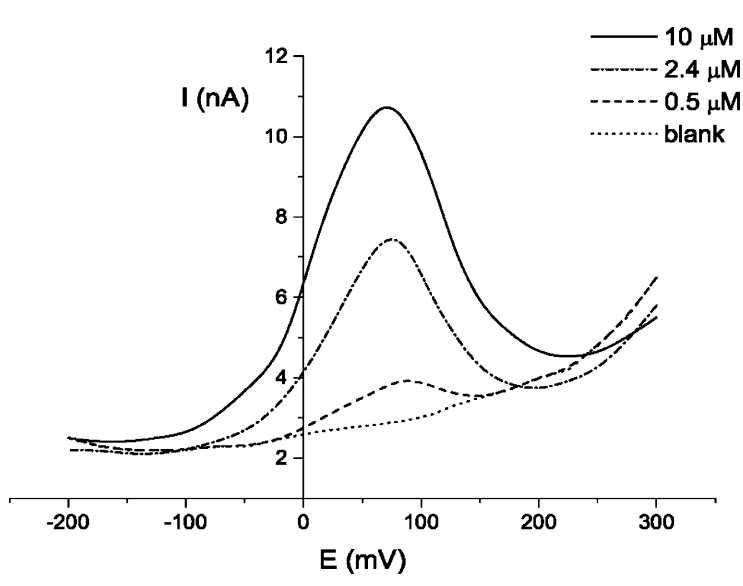

Fig. 3. Differential pulse voltammograms recorded at a NEE in solutions containing increasing cyt $c$ concentrations, as indicated in the figure. Experimental parameters: scan rate $5 \mathrm{mV} \mathrm{s}^{-1}$, pulse height $25 \mathrm{mV}$, initial potential $+300 \mathrm{mV}$ and final potential $-200 \mathrm{mV}$.

published. This was shown to be the case at glassy carbon if proper supporting electrolyte, aged solutions, etc. were used $[25,26]$. Direct signals were observed also on heated wire gold ultramicroelectrodes [27] and using electrodes coated with Au nanoparticles [28,29].

Fig. 3 shows the DPV signals recorded directly at a NEE in solutions containing trace (micro- and submicromolar) cyt $c$ concentrations. A well resolved DPV peak is detected at $70 \mathrm{mV}$, even in $0.5 \mu \mathrm{M}$ cyt $c$ solutions. The peak potential value as well as the dependence of the peak current on cyt $c$ concentration indicate that the observed signal is due to the one-electron electrochemical reduction of Fe(III) of the protein heme-group [18], according to reaction (7):

[cyt $c$-Fe(III)] $+\mathrm{e} \rightarrow$ [cyt $c$-Fe(II)]

The half width of the peak is about $110 \mathrm{mV}$, that indicates a quasi-reversible one electron transfer. Detection limit of cyt $c$ at NEEs in the absence of any promoter is $0.03 \mu \mathrm{M}$, which is equal to the DL determined at NEE in the presence of $4,4^{\prime}$-bipy as promoter. Such DL values are more than one order of magnitude lower than DL at gold macroelectrode in the same experimental conditions [15].

These data show the possibility to observe the direct electrochemistry of cyt $c$ at submicromolar solution concentrations even in the absence of added promoter by using DPV at NEEs.

The mechanistic analysis of the electrochemistry of cyt $c$ at NEEs is out of the goals of the present investigation which is aimed specifically to show analytical peculiarities of NEEs for traces electroanalysis, in particular when NEEs and DPV are used together. Such aspects are examined in details in a forthcoming paper [16].

\section{Conclusions}

It is shown that improvements in signal/background current ratios at NEEs are independent on the total geometric area of the ensemble; this is true if the fractional area is kept constant and if the dimensions of the ensemble are lowered to a size still large enough to contain a large number of nanoelements (e.g. our NEE with $A_{\text {geom }}$ of $0.005 \mathrm{~cm}^{2}$ contains $4.8 \times 10^{6}$ nanoelectrodes). Note that NEEs warranty such an independence on the ensemble size for overall geometric areas much lower than those required for achieving comparable results with arrays of micrometer sized electrodes [9]. This is particularly attractive when thinking to apply the advantages of the use of arrays/ensembles of microelectrodes to analyses in samples of very small volume.

NEEs show improved detection limits with respect to conventional electrodes also when DPV is used as the detection technique, giving extended flat background currents. This is the case both for reversible redox probes as $\mathrm{FA}^{+}$, and also for electroactive analytes more difficult to detect electrochemically such as cyt $c$. The use of NEEs and DPV allows the detection of submicromolar concentrations of redox molecules without the need of using time consuming preconcentration steps.

\section{Acknowledgements}

Financial support from MIUR (Rome) is acknowledged.

\section{References}

[1] C.R. Martin, in: A.J. Bard, I. Rubinstein (Eds.), Electroanalytical Chemistry, vol. 21, Marcel Dekker, New York, 1999, pp. 1-74.

[2] P. Ugo, L.M. Moretto, F. Vezzà, Chem. Phys. Chem. 3 (2002) 917.

[3] V.P. Menon, C.R. Martin, Anal. Chem. 67 (1995) 1920.

[4] J.F. Cheng, L.D. Whiteley, C.R. Martin, Anal. Chem. 61 (1989) 762.

[5] J.C. Hulteen, V.P. Menon, C.R. Martin, J. Chem. Soc., Faraday Trans 92 (1996) 4029.

[6] S. Szunerits, D.R. Wait, Anal. Chem. 74 (2002) 1718.

[7] C. Amatore, J.M. Saveant, D. Tessier, J. Electroanal. Chem. 147 (1983) 39.

[8] B. Brunetti, P. Ugo, L.M. Moretto, C.R. Martin, J. Electroanal. Chem. 491 (2000) 166.

[9] H.J. Lee, C. Beriet, R. Ferrigno, H.H. Girault, J. Electroanal. Chem. $502(2001) 138$.

[10] P. Ugo, L.M. Moretto, S. Bellomi, V.P. Menon, C.R. Martin, Anal. Chem. 68 (1996) 4160.

[11] A. Lombardo, T.I. Bieber, J. Chem. Educ. 60 (1983) 1080.

[12] V. Massey, Biochim. Biophys. Acta 34 (1959) 255.

[13] P. Ugo, V. Zangrando, L.M. Moretto, B. Brunetti, Biosens. Bioelectron. 17 (2002) 479.

[14] F.N. Büchi, A.M. Bond, J. Electroanal. Chem. 314 (1991) 191.

[15] A. Szucs, G.D. Hitchens, J.O’M. Bockris, Electrochim. Acta 37 (1992) 403.

[16] P. Ugo, N. Pepe, L.M. Moretto, M. Battagliarin, J. Electroanal. Chem. 560 (2003) 51.

[17] A.J. Bard, L.R. Faulkner, Electrochemical Methods, second ed., Wiley, New York, 2000.

[18] M.J. Eddowes, H.A.O. Hill, J. Chem. Soc., Chem. Commun. (1977) 771. 
[19] P.M. Allen, H.A.O. Hill, N.J. Walton, J. Electroanal. Chem. 178 (1984) 69.

[20] I. Taniguchi, K. Toyosawa, H. Yamaguchi, K. Yasukuoki, J. Chem. Soc., Chem. Commun. (1982) 1032.

[21] I. Taniguchi, M. Iseki, T. Eto, K. Toyosawa, H. Yamaguchi, K. Yasukouchi, Bioelectrochem. Bioenerg. 13 (1984) 373.

[22] A. Avila, B.W. Gregory, K. Niki, T.M. Cotton, J. Phys. Chem. B 104 (2000) 2759.

[23] J.M. Cooper, K.R. Greenough, C.J. McNeil, J. Electroanal. Chem. 347 (1993) 267.
[24] E. Lojou, P. Luciano, S. Nitsche, P. Bianco, Electrochim. Acta 44 (1999) 3341.

[25] A. Szücs, M. Novák, J. Electroanal. Chem. 383 (1995) 75.

[26] A. Szücs, M. Novák, J. Electroanal. Chem. 384 (1995) 47.

[27] T. Voss, P. Grundler, C.M.A. Brett, A.M. Oliveira Brett, J. Pharm. Biomed. Anal. 19 (1999) 127.

[28] K.R. Brown, A.P. Fox, M. Natan, J. Am. Chem. Soc. 118 (1996) 1154.

[29] H. Ju, S. Liu, B. Ge, F. Lisdat, F.W. Scheller, Electroanalysis 14 (2002) 141. 